\title{
SOME RECENT INVESTIGATIONS IN THE THEORY OF HYDRODYNAMIC STABILITY*
}

\author{
BY \\ J. R. FOOTE (Iowa State College) \\ AND \\ C. C. LIN (Massachusetts Institute of Technology)
}

1. Introduction. In most of the existing investigations of the stability of two-dimensional parallel flows [1], two essential restrictions have been imposed: (1) at least one solid boundary is present, and (2) the velocity distribution is a monotone curve. It has been possible to treat the case of symmetrical velocity distributions through a channel by imposing the boundary condition at the axis of symmetry and therefore fulfilling the above conditions.

There is a large class of parallel flows where no solid boundary is present. These are the problems of "free boundary layers", such as the flow field in the jet, the wake, and the mixing region between two streams. It may be expected at once from physical considerations that the influence of the "viscous solutions" of the disturbance equation should not occur, because their influence is known to be chiefly of the nature of a boundary layer phenomenon in the immediate neighborhood of the solid boundary. In the case of the mixing region between two streams, this fact can also be easily seen mathematically. The exponential nature of the "viscous solutions" is well known, and this behavior is definitely incompatible with the condition that the disturbances must have finite amplitudes at large distances. The effect of viscosity must therefore be found from the second order approximation of the "inviscid solutions." The viscous influence is therefore restricted to the immediate neighborhood of the critical layer, and the general stability characteristics of such flows should therefore be very much different from those previously investigated.

With these ideas in mind, Lin suggested the investigation of the stability characteristics of such flows to M. Lessen [2] (using numerical methods) and to P. Chiarulli [3] (using analytical methods). Lessen made detailed calculations for one profile with the help of Dr. L. H. Thomas of I. B. M. of New York City. The results show that the effect of viscosity is always to stabilize the flow even for very large Reynolds numbers, contrary to Heisenberg's criterion for cases with solid boundary. The critical Reynolds number is found to be very low, as may be expected from the presence of the point of inflection in the velocity distribution and the limited extent of the effect of viscosity. Chiarulli obtained results for general velocity distributions of the mixing type. It appears from his analysis that the stabilizing effect of viscosity is probably a general characteristic of such flows.

For jets and wakes it is not obvious that the above discussions apply. In the case of symmetrical jets and wakes, one may apply the usual type of analysis and reach the above conclusions concerning the origin of the viscous effects (see Sec. 2). The case of unsymmetrical distributions is in general more difficult, because there are usually

\footnotetext{
*Received Aug. 21, 1949. A part of this paper is based on a thesis submitted by J. R. Foote in partial fulfillment of the requirements for the degree of Doctor of Philosophy at the Massachusetts Institute of Technology.
} 
two critical points in the flow field. The purpose of the present paper is to clarify the nature of the asymptotic solutions in cases with two critical points. It will be shown by a slight extension of some work of Wasow [4] that to preserve the validity of one asymptotic expression for the solutions, a path must be taken below the critical point in the region where the slope of the velocity curve is positive, and above the critical point in the region with negative slope. Application of this result to the cases of unsymmetrical jets and wakes again shows that the "viscous solutions" must be rejected and that the effect of viscosity must come from the second order approximation of the "inviscid solutions".

The remaining part of the paper is devoted to an investigation of the inviscid approximation, with special reference to the stability of jets, wakes, and the zonal winds in the atmosphere. It is shown that, in all cases, a necessary and sufficient condition for the existence of (non-trivial) neutral and self-excited disturbances is the occurrence of the maximum or minimum of vorticity. This is true even in the case of zonal winds where the point of inflection of the profile does not have the usual significance, and the usual proof of sufficiency does not apply.

It is further shown that for unsymmetrical velocity distributions the neutral disturbance may give rise to a Reynolds shear which is opposite in sign to the velocity gradient of the mean flow. In other words, these ordered oscillations, unlike fully developed turbulent motions, can transmit momentum from a region of lower mean velocity to a region of higher mean velocity

2. Asymptotic solutions and boundary value problems. The derivation of the linearized stability equation

$$
(w-c)\left(\varphi^{\prime \prime}-\alpha^{2} \varphi\right)-w^{\prime \prime} \varphi=-\frac{i}{\alpha R}\left(\varphi^{\mathrm{IV}}-2 \alpha^{2} \varphi^{\prime \prime}+\alpha^{4} \varphi\right),
$$

and the explanation of symbols can be found in [1]. We consider jet profiles extending to infinity in both directions. Boundary conditions for these profiles may be written

$$
\begin{gathered}
\lim _{y \rightarrow \infty}\left[\frac{\varphi^{\prime}(y)}{\varphi(y)}\right]=\mp \alpha, \\
\lim _{y \rightarrow+\infty} u=0, \quad \lim _{y \rightarrow+\infty} v=0 .
\end{gathered}
$$

For the symmetric profile one may use one of these conditions together with one of the following:

$$
\begin{aligned}
\varphi(0)=0, & \text { if } \varphi(y) \text { is odd, } \\
\varphi^{\prime}(0)=0, & \text { if } \varphi(y) \text { is even. }
\end{aligned}
$$

It has been shown by Wasow [4] that the asymptotic solutions of (1) may be obtained by formal expansions. In Sec. 6 we shall extend his result slightly and discuss how these solutions can be applied to profiles having more than one critical point. It will be shown that there are asymptotic solutions valid in a region defined as follows. The critical points where $w(y)=c$ shall be denoted by $y_{c}$ and $y_{0}$, with $\operatorname{Re}\left[w^{\prime}\left(y_{c}\right)\right]>0$ and $\operatorname{Re}\left[w^{\prime}\left(y_{0}\right)\right]<0$. Through the point $y_{c}$ there are three lines $C_{k}, k=1,2,3$, defined by

$$
\operatorname{Re} Q_{c}(y)=\operatorname{Re}\left\{\int_{y_{c}}^{y}[i(w-c)]^{1 / 2} d y\right\}=0
$$


with three similar lines $C_{k}^{*}, k=1,2,3$, through $y_{0}$ (Fig. 1). Consider a path $\Gamma$ extending

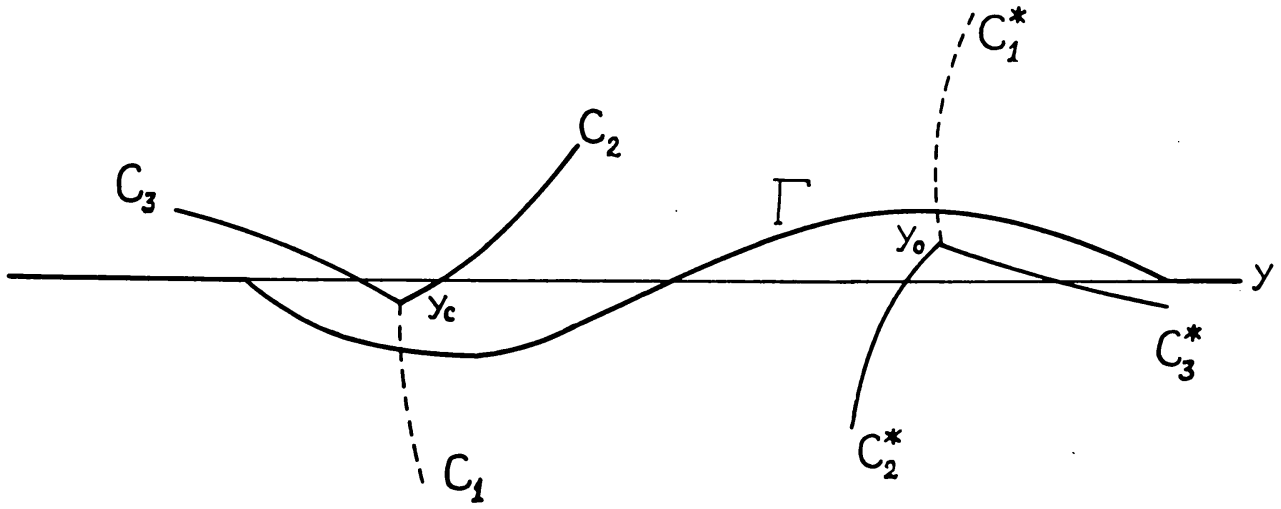

FIG. 1.

from $y=-\infty$ to $y=+\infty$, and crossing only one each of the lines $C_{k}$ and $C_{k}^{*}$. A neighborhood of such a path $\Gamma$ is a region of validity of the asymptotic solutions.

The explicit expansions of the solutions are:

$$
\begin{aligned}
& \varphi_{1}=\varphi_{1}^{(0)}+\varphi_{1}^{(1)}(\alpha R)^{-1}+\varphi_{1}^{(2)}(\alpha R)^{-2}+\cdots \\
& \varphi_{2}=\varphi_{2}^{(0)}+\varphi_{2}^{(1)}(\alpha R)^{-1}+\varphi_{2}^{(2)}(\alpha R)^{-2}+\cdots \\
& \varphi_{3}=\exp \left[-(\alpha R)^{1 / 2} Q_{c}(y)\right]\left\{(w-c)^{-5 / 4}+O(\alpha R)^{-1 / 2}\right\} \\
& \varphi_{4}=\exp \left[+(\alpha R)^{1 / 2} Q_{c}(y)\right]\left\{(w-c)^{-5 / 4}+O(\alpha R)^{-1 / 2}\right\}
\end{aligned}
$$

The notation agrees with that used in [1]. The solutions $\varphi_{3}$ and $\varphi_{4}$ respectively become infinite exponentially as $y \rightarrow-\infty$ and $+\infty$. It is then clear that $c_{3}=c_{4}=0$ in the general solution

$$
\varphi=c_{1} \varphi_{1}+c_{2} \varphi_{2}+c_{3} \varphi_{3}+c_{4} \varphi_{4}
$$

if we apply the boundary conditions at $y= \pm \infty$, for either the symmetric or the unsymmetric profile. Thus the effect of viscosity would appear to enter only through the higher approximations for $\varphi_{1}, \varphi_{2}$. This should be discussed further for the symmetric profile since $(2 \mathrm{a})$ or $(2 \mathrm{~b})$ may be used with one of the conditions (2). Then we are using only half the profile, so that $\varphi_{4}$ does not diverge and may be included:

$$
\varphi=c_{1} \varphi_{1}+c_{2} \varphi_{2}+c_{4} \varphi_{4} .
$$

For case (2a) the determinantal eigenvalue condition is then

$$
\left|\begin{array}{ccc}
\varphi_{11}^{\prime}-\alpha \varphi_{11} & \varphi_{21}^{\prime}-\alpha \varphi_{21} & 0 \\
\varphi_{12} & \varphi_{22} & \varphi_{42} \\
\varphi_{12}^{\prime \prime} & \varphi_{22}^{\prime \prime} & \varphi_{42}^{\prime \prime}
\end{array}\right|=0,
$$

where we have put $y_{1}=-\infty, y_{2}=0$, and $\varphi_{11}^{\prime}=\varphi_{1}^{\prime}\left(y_{1}\right), \varphi_{12}=\varphi_{1}\left(y_{2}\right)$, and so on. How- 
ever, we shall show that the viscous effects are still not introduced by the "viscous solutions."

Suppose $\Phi=k_{1} \varphi_{1}+k_{2} \varphi_{2}$ is a solution satisfying the inviscid equation

$$
(w-c)\left(\varphi^{\prime \prime}-\alpha^{2} \varphi\right)-w^{\prime \prime} \varphi=0
$$

to the first approximation and satisfying the boundary condition at $y_{1}$. Taking this linear combination of the first two columns in (5) and replacing the first column by the result and simplifying, we obtain the eigenvalue condition as

$$
\Phi_{2}=\Phi_{2}^{\prime \prime} \frac{\varphi_{42}}{\varphi_{42}^{\prime \prime}}
$$

Using (4) we find expressions for $\Phi_{2}$ and $\varphi_{42} / \varphi_{42}^{\prime \prime}$. Then (7) becomes

$$
\begin{aligned}
\Phi_{2}^{(0)} & +\frac{\Phi_{2}^{(1)}}{\alpha R}+\cdots \\
& =\left[\Phi_{2}^{(0) \prime}+\frac{\Phi_{2}^{(1) \prime \prime}}{\alpha R}+\cdots\right] \frac{1}{i(w-c)_{2} \alpha R}[1+\cdots] .
\end{aligned}
$$

Neglecting terms of order $0(\alpha R)^{-1}$, (8) yields:

$$
\Phi_{2}^{(0)}=0 \text {. }
$$

Then it follows from (6) that $\Phi_{2}^{(0)}$ " also vanishes. If we now include terms of order $O(\alpha R)^{-1},(8)$ yields:

$$
\Phi_{2}^{(0)}+\frac{\Phi_{2}^{(1)}}{\alpha R}=0,
$$

and again there is no contribution to viscosity from $\varphi_{4}$. This process can be continued with the aid of the recursion formulae for $\varphi_{1}$ and $\varphi_{2}[1]$.

We can perform the same steps with case $(2 \mathrm{~b})$ and find the condition corresponding to $(7)$ as

$$
\Phi_{2}^{\prime}=\Phi_{2}^{\prime \prime \prime} \frac{\varphi_{42}^{\prime}}{\varphi_{42}^{\prime \prime \prime}}
$$

Here $\varphi_{42}^{\prime} / \varphi_{42}^{\prime \prime \prime}$ has the same form as in (8), but (6) gives

$$
\Phi_{2}^{(0) \prime \prime \prime}=\left(\frac{w^{\prime \prime}}{w-c}\right)_{2}^{\prime} \Phi_{2}^{(0)}+\left(\alpha^{2}+\frac{w^{\prime \prime}}{w-c}\right)_{2} \Phi_{2}^{(0)} .
$$

Now $w^{\prime \prime}$ and $w-c$ are even functions of $y$, so their ratio is even and the derivative shown is odd and hence zero at $y_{2}=0$. Hence $\Phi_{2}^{(0)}$ "' vanishes with $\Phi_{2}^{(0)}$ ', and the calculations proceed in exactly the same way. Therefore we conclude that the effect of viscosity enters the stability problem for the jet profile (symmetric or unsymmetric) only through the higher approximations to the "inviscid solutions" of the stability equation (1). This is an extension of the conclusion reached in the infinite jet (mixing problem) and is in marked contrast to other stability problems previously investigated.

We note the following important facts: (a) the only usable solutions of (1) tend, as $\alpha R \rightarrow \infty$, to a fundamental system of (6), which may be obtained from (1) by formally passing to the limit of infinitely large Reynolds number; (b) The stability problem for 
(1) (expressed in the eigenvalue condition) tends to the stability problem of (6) as $\alpha R \rightarrow \infty$. These facts give importance to a study of the inviscid equation.

3. The inviscid equation. The inviscid equation (8) is essentially the vorticity equation for a perfect fluid. The term $-w^{\prime \prime}(y)$ is known to represent the gradient of vorticity [1]. In other problems of stability of parallel flows (see Sec. 5), the gradient of vorticity also enters the problem, but it is not necessarily related to the main velocity distribution $w(y)$ in this simple manner. We shall therefore consider the equation

$$
[w(y)-c]\left(\varphi^{\prime \prime}-\alpha^{2} \varphi\right)+g(y) \varphi=0,
$$

where $g(y)$ is the gradient of vorticity, and is to be replaced by $-w^{\prime \prime}(y)$ in the simple case. It is obvious that the results of Sec. 2 are applicable without modification, because they depend only on the coefficient $w-c$ of $\varphi^{\prime \prime}$.

Solution of (9) by means of power series gives the fundamental system*

$$
\begin{aligned}
& \varphi_{1}(y)=\left(y-y_{c}\right)+\cdots \\
& \varphi_{2}(y)=1+\cdots-\frac{g\left(y_{c}\right)}{w^{\prime}\left(y_{c}\right)} \varphi_{1} \log \left(y-y_{c}\right)
\end{aligned}
$$

in the neighborhood of $y=y_{c}$, and a similar system in the neighborhood of the critical point $y=y_{0}$. The only difference lies in the path for the determination of the logarithmic function. The path is to be taken below the point $y_{c}$, but above the point $y_{0}$ (see Fig. 1). Thus, in the case of self-excited disturbances, the inviscid equation holds all along the real axis. But in the case of damped disturbances it fails over portions of the real axis in the neighborhood of $y_{c}$ and $y_{0}$. The general solution of (9) in the neighborhood of $y_{c}$ is

$$
\varphi=A \varphi_{1}+B \varphi_{2}=A \varphi_{1}+B \varphi_{10}-B \frac{g_{c}}{w_{c}^{\prime}} \varphi_{1} \log \left(y-y_{c}\right),
$$

where $\varphi_{10}$ is the power series part of $\varphi_{2}$ and $A$ and $B$ are complex constants.

It is found most convenient to study the inviscid equation by examining the Reynolds shear stress, as was first done by Tollmien [5]. We shall now generalize his analysis from several points of view and then apply the results to various types of velocity distributions.

Assuming the existence of the solutions, we shall establish three general relations. The actual existence problem will be discussed later.

(i) The Reynolds shear stress is

$$
\tau=-\rho \overline{u^{\prime} v^{\prime}}=\rho \frac{\alpha^{2}}{2} e^{c_{i} t} W\left(\varphi_{r}, \varphi_{i}\right),
$$

where $\rho$ is the density of the fluid, $u^{\prime}$ and $v^{\prime}$ are the real disturbance velocities, the averaging process is taken with respect to $x$, and $W\left(\varphi_{r}, \varphi_{i}\right)$ is the Wronskian $\varphi_{r} \varphi_{i}^{\prime}-\varphi_{i} \varphi_{r}^{\prime}$ of the real and imaginary parts $\varphi_{r}(y)$ and $\varphi_{i}(y)$ of $\varphi(y)$ for real values of $y$. Tollmien discussed this relation only for neutral solutions with $c_{i}=0$.

(ii) The rate of change of the Reynolds shear stress $\tau$ along the real axis is given by

$$
\frac{d \tau}{d y}=-\rho \frac{\alpha^{2}}{2} e^{2 c_{i t} t} c_{i} g|\varphi|^{2} /|w-c|^{2} .
$$

*This is a slight generalization of Tollmien's result [5]. 
It is obvious that both the above results are applicable only for parts of the real axis where $\varphi$ is regular.

(iii) In the case of the neutral disturbance, there is a discontinuity of the Reynolds shear at the critical point of amount

$$
[\tau]=\tau\left(y_{c}+0\right)-\tau\left(y_{c}-0\right)=-\frac{\alpha^{2}}{2} \pi \rho\left|\varphi_{c}\right|^{2} g_{c} /\left|w_{c}^{\prime}\right| .
$$

All these results can be obtained by straightforward calculations. The real disturbance velocities $u^{\prime}$ and $v^{\prime}$ (with real values of $y$ ) are given by

$$
u^{\prime}=\operatorname{Re}\left(\psi_{y}^{\prime}\right), \quad v^{\prime}=\operatorname{Re}\left(-\psi_{x}^{\prime}\right),
$$

with the disturbance stream function

$$
\psi^{\prime}(x, y, t)=\varphi(y) e^{i \alpha(x-c t)} .
$$

Thus, the average

$$
\overline{u^{\prime} v^{\prime}}=\lim _{x \rightarrow \infty} \frac{1}{x} \int_{0}^{x} u^{\prime} v^{\prime} d x
$$

is easily seen to be

$$
\overline{u^{\prime} v^{\prime}}=\frac{\alpha^{2}}{2} e^{2 c_{i} t}\left(\varphi_{i} \varphi_{r}^{\prime}-\varphi_{r} \varphi_{i}^{\prime}\right),
$$

from which (12) follows immediately. One may also write

$$
\varphi_{i} \varphi_{r}^{\prime}-\varphi_{r} \varphi_{i}^{\prime}=\frac{1}{2 i}\left(\varphi \bar{\varphi}^{\prime}-\bar{\varphi} \varphi^{\prime}\right),
$$

so that

$$
W\left(\varphi_{r}, \varphi_{i}\right)=\frac{i}{2}\left(\bar{\varphi} \bar{\varphi}^{\prime}-\bar{\varphi} \varphi^{\prime}\right) .
$$

It should be noted that the conjugate signs are formal conjugates for real values of $y$, i.e.,

$$
\bar{\varphi}=\varphi_{r}(y)-i \varphi_{i}(y), \quad \bar{\varphi}^{\prime}=\varphi_{r}^{\prime}(y)-i \varphi_{i}^{\prime}(y) .
$$

From (15) one can easily derive (13) as follows. Rewriting (9) in the form

$$
\varphi^{\prime \prime}-\alpha^{2} \varphi+\frac{g(y)}{w(y)-c} \varphi=0,
$$

multiplying it by $\bar{\varphi}$ and subtracting from it the complex conjugate equation, one obtains

$$
\frac{d}{d y}\left(\varphi^{\prime} \bar{\varphi}-\bar{\varphi}^{\prime} \varphi\right)=-\frac{|\varphi|^{2}}{|w-c|^{2}} 2 c_{i} g_{i},
$$

or

$$
\frac{d W}{d y}=-\frac{|\varphi|^{2}}{|w-c|^{2}} c_{i} g .
$$

By combining this relation with (12), the relation (13) is obtained at once. 
To obtain (14) it is only necessary to calculate the jump of $W$, which in turn is determined from the jump of $\varphi^{\prime}$, since $\varphi$ is continuous across the singular point. Thus, from (15),

$$
[W]=\frac{i}{2}\left\{\varphi \overline{\varphi\left[\varphi^{\prime}\right]}-\bar{\varphi}\left[\varphi^{\prime}\right]\right\}, \quad \text { for } y=y_{c} .
$$

From (10) and (11) it is clear that

$$
\varphi_{c}=B, \quad\left[\varphi^{\prime}\right]=-B \frac{g_{c}}{\left|w_{c}^{\prime}\right|} i \pi,
$$

since the angle of $y-y_{c}$ increases by $\pi$ when $y$ passes from $y_{c}-0$ to $y_{c}+0$ if $w_{c}^{\prime}>0$ and decreases if $w_{c}^{\prime}<0^{*}$. Thus,

$$
[W]=-\pi \frac{g_{c}}{\left|w_{c}^{\prime}\right|}\left|\varphi_{c}\right|^{2} .
$$

By combining (17) and (12), (14) follows at once.

We shall now prove the following theorem giving a necessary condition for the existence of self-excited and neutral disturbances. Theorems on sufficiency of conditions will be discussed in the next sections.

THEOREM I. If the disturbance must satisfy homogeneous boundary conditions at each of two points $y_{1}$ and $y_{2}$, the condition $g\left(y_{s}\right)=0$ for some $y_{s}$ in the interval $\left(y_{1}, y_{2}\right)$ is necessary for the existence of self-excited disturbances, and of neutral disturbances with $w=c$ at some interior point of the interval $\left(y_{1}, y_{2}\right)$.

In the case $g(y)=-w^{\prime \prime}(y)$, neutral disturbances with $w \neq c$ in the closed interval $\left(y_{1}, y_{2}\right)$ are also excluded by the condition $g(y) \neq 0$ except possibly the trivial solution with $\alpha=0, \varphi=(w-c)$, (which must be examined with the boundary conditions).

PROOF: The first part can be easily proved by examining the variation of $\tau$ for real values of $y$. At the points $y_{1}$ and $y_{2}, \tau$ vanishes. If a self-excited disturbance exists, and $g(y)$ does not vanish between $y_{1}$ and $y_{2}$, then (13) shows that $\tau$ varies monotonically in between, and hence cannot possibly fulfill the boundary conditions.

For a neutral disturbance, $\tau$ is a constant except for the jumps given by (14). To satisfy the boundary conditions, one must have

$$
\sum[\tau]=0,
$$

summation being taken for all the jumps. Since $w_{\min }<c<w_{\max }$, there is at least one jump. If there is only one, we see at once that $g_{c}=0$, or alternatively $\varphi_{c}=0$. We must show that the second alternative is impossible. Now from (10) and (11), it is clear that $\varphi_{c}=0$ implies that

$$
\varphi=(w-c) f,
$$

where $f$ is a regular function. If we now multiply (9a) by $\bar{\varphi}$ and integrate between $y_{1}$ and $y_{c}$, we obtain

$$
\int_{\nu_{1}}^{\nu_{2}}\left\{\left|\varphi^{\prime}\right|^{2}+\alpha^{2}|\varphi|^{2}\right\} d y=\int_{\nu_{1}}^{y_{c}} g(w-c)|f|^{2} d y \geq 0 .
$$

\footnotetext{
*The result therefore also applies to the neighborhood of $y_{0}$.
} 
Similarly,

$$
\int_{y_{c}}^{y_{2}} g(w-c) \mid f \dot{\dagger}^{2} d y \geq 0 .
$$

Since $w-c$ definitely has opposite signs in the two intervals $\left(y_{1}, y_{c}\right)$ and $\left(y_{c}, y_{2}\right)$, it is obvious that we have a contradiction if the sign of $g$ remains unchanged.

If there is more than one point where $w=c$, either (i) all the $\varphi_{c}$ 's are zero, or (ii) some of the $g_{c}$ 's vanish or have opposite signs. The first case can be excluded by an argument similar to the above. The other case insures the vanishing of $g(y)$ within $\left(y_{1}, y_{2}\right)$.

To establish the second part of the theorem, let us note that we again have (19) when $w \neq c$ in $\left(y_{1}, y_{2}\right)$. We may rewrite (9) as

$$
\frac{d}{d y}\left\{(w-c)^{2} f^{\prime}\right\}-\alpha^{2}(w-c)^{2} f+\left(w^{\prime \prime}+g\right)(w-c) f=0 .
$$

Multiplying this equation by $\bar{f}$ and integrating between $y_{1}$ and $y_{2}$, we obtain

$$
\int_{\nu_{1}}^{y_{2}}(w-c)^{2}\left\{\left|f^{\prime}\right|^{2}+\alpha^{2}|f|^{2}\right\} d y=\int_{\nu_{1}}^{y_{2}}\left(w^{\prime \prime}+g\right)(w-c)|f|^{2} d y .
$$

This relation does not allow much to be inferred, except in the important case $g=-w^{\prime \prime}$. In this case, we must have

$$
\alpha=0, \quad f=\text { const., }
$$

which proves our statement. This method of proof is well-known [6], but we include the details here to show why it does not work for general $g(y)$.

4. Wakes and jets. We shall now investigate more closely several typical problems. In this section, we shall study the stability of two-dimensional wakes and jets, symmetrical and unsymmetrical. For these problems, $g(y)=-w^{\prime \prime}(y)$. In the next section, we shall study the stability of zonal winds over a surface of revolution.

(a) Symmetrical jets. In this case, the neutral disturbance can occur only when the wave velocity $c$ is equal to the flow velocity at the point of inflection, except for the trivial case given in Theorem I. The Reynolds shear is zero everywhere, and there is no transfer of energy between the main flow and the disturbance.

For self-excited oscillations, (13) leads to a shear distribution shown in Fig. 2. One notes that $\tau>0$ wherever $w^{\prime}(y)>0$. The energy is transferred from the main flow to the disturbance by the Reynolds shear, as it does in fully developed turbulent motion. For damped disturbances, the inviscid theory is applicable only to certain parts of the flow field. But (13) shows clearly that there will be regions where the shear $\tau$ has an opposite sign to the velocity gradient $w^{\prime}(y)$; the damped disturbance feeds energy into the main flow in these regions.

We shall see that for unsymmetrical jets, $\tau$ and $w^{\prime}(y)$ may have opposite signs even for the neutral disturbance.

We have yet to prove the existence of neutral and self-excited disturbances. This may be done in the usual manner for the even solution satisfying the boundary conditions $\varphi^{\prime}(0)=0$ and $\varphi \rightarrow 0$ as $y \rightarrow 0$. The proof given by Friedrichs [6] can be adopted without change, since $w(y)$ satisfies the boundary conditions required of $\varphi(y)$. The existence of self-excited disturbances can be proved by following the method first used 
by Tollmien [5] and slightly extended by Lin [1, Part 2]. The convergence of the integrals involved can be easily verified.

The trivial solution $\varphi=w-c$ for $\alpha=0$ is valid for all values of $c$, if one merely requires that the disturbance vanishes at infinity, for $\left(u^{\prime}, v^{\prime}\right)$ are proportional to $\left(\alpha \varphi, \varphi^{\prime}\right)$.

(b) Unsymmetric jets. The existence of neutral and self-excited disturbances for such cases can best be seen from a continuity argument. Thus, if one decomposes the velocity distribution $w(y)$ into an even part $w_{1}(y)$ and an odd part $w_{2}(y)$, one may consider it as a member of the family $w_{1}(y)+\epsilon w_{2}(y)$. When $\epsilon$ is zero, self-excited disturbances exist for $0<\alpha<\alpha_{s}$, say. When $\epsilon$ changes, the eigenvalue must change in a continuous

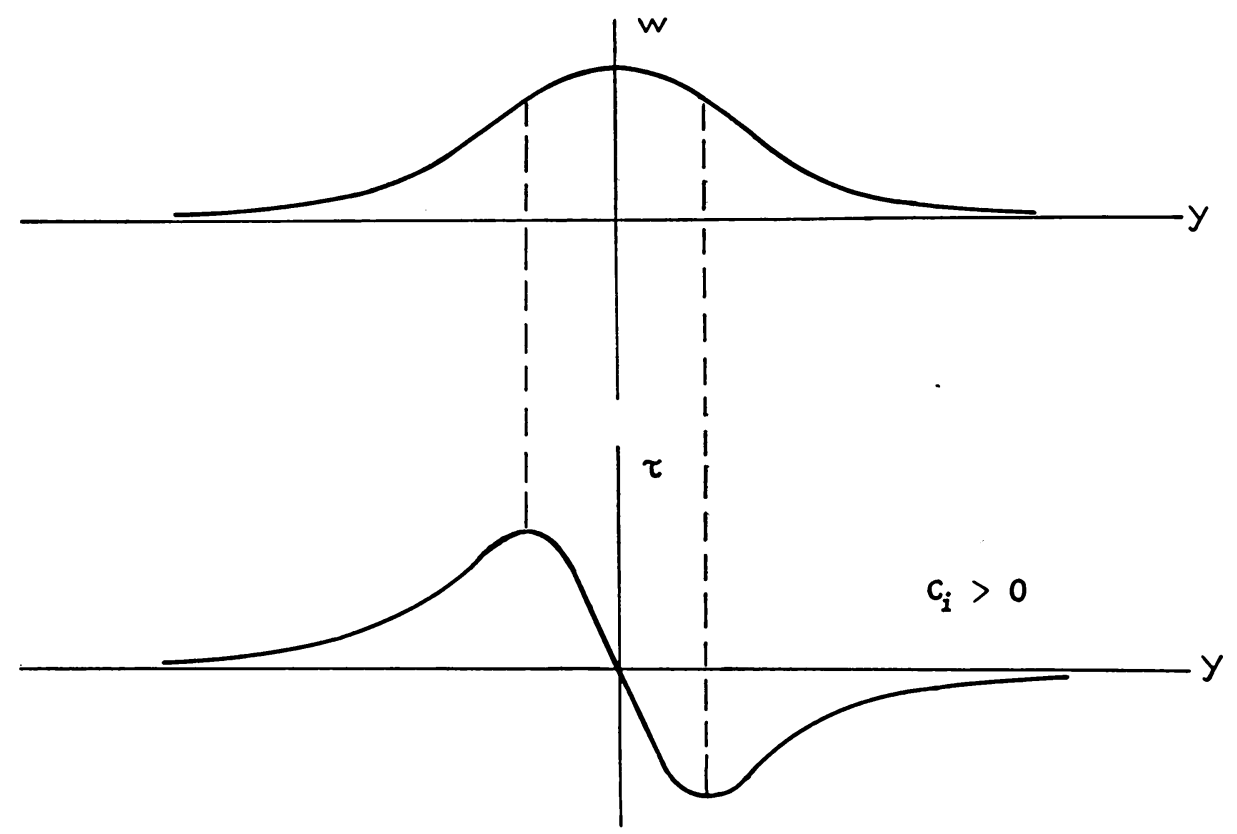

FIG. 2.

manner. Thus, there would still be a finite range of $\alpha$ for which the motion is self-excited and the end points of which correspond to neutral disturbances. But the range might shrink to zero when $\epsilon$ is fairly large. Thus, at least for velocity distributions not far from the symmetrical case, we have self-excited and neutral disturbances. One notes, however, that $\varphi=(w-c)$ is always a solution for $\alpha=0$.

The shear stress distribution is very interesting in the present case. For definiteness, let the points where $w^{\prime \prime}=0$ be denoted by $y_{s}$ and $y_{t}$ with $y_{s}<y_{t}$ and $w_{s}>w_{t}$. For the neutral disturbance, it is then necessary that

$$
w_{s}>c>w_{t},
$$

because this is the only way to make $w_{s}^{\prime \prime} w_{t}^{\prime \prime}<0$. (See (14) and (18)). The distribution of stress $\tau$ is as shown in Fig. 3 (dotted). The stress suddenly jumps from zero to a finite value at $y_{c}$, stays constant, and then jumps back to zero at $y_{0}$. For the self-excited disturbance, (13) gives the distribution shown by the full line of Fig. 3. The maximum 
stress occurs at $y_{\mathrm{s}}$ and the minimum at $y_{t}$. One notes that in either case there is a part where $\tau$ and $w^{\prime}(y)$ are of opposite signs. The net transfer of energy in the neutral case is, however, zero, because

$$
\int_{\nu_{e}}^{y_{0}} \tau w^{\prime}(y) d y=\tau \int_{\nu_{e}}^{\nu_{0}} w^{\prime}(y) d y=0 .
$$

If $w_{s}<w_{t}$, the sign of $\tau$ is essentially negative. In either case, the shear stress in the case of the neutral disturbance transfers momentum from the part with lower velocity at the point of inflection to the part with higher velocity.

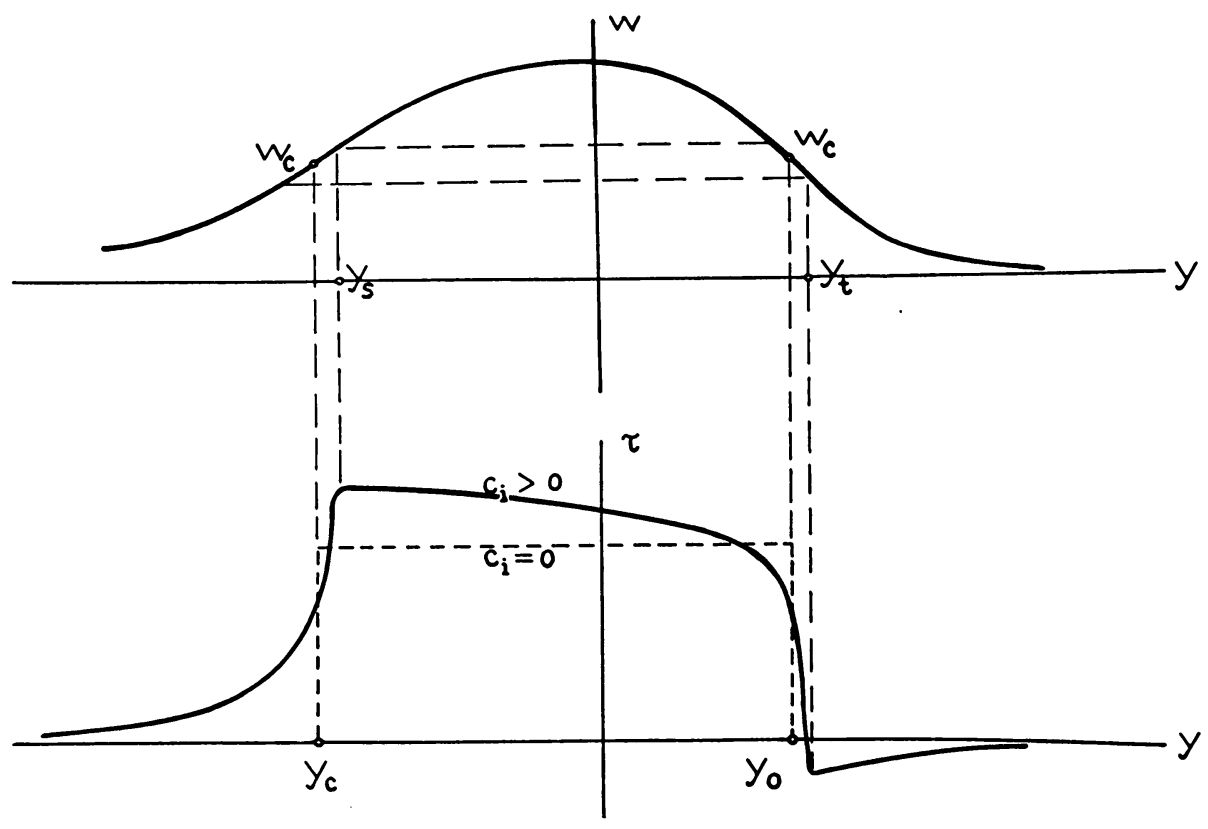

FIG. 3.

5. Instability of zonal winds*. We shall now apply our results to the motion of a thin layer of fluid over the surface of a spinning body of revolution, say, the earth. These results may be applied to stability of the zonal winds in the atmosphere. Discussions of such problems have been made by H. L. Kuo [8], largely by considering plane motions. With the help of present results, we can make a more thorough investigation of the problem.

Let us take an orthogonal coordinate system $(\varphi, \sigma, z)$, where $\varphi$ is the longitude, $\sigma$ is the distance from the equator along a meridian positive towards the north pole, and $z$ is the distance perpendicular to the surface. Consider two-dimensional motion of a thin layer of fluid in the surfaces of constant $z$. We may put $z=0$ throughout the investigation. Let $u$ and $v$ be the linear velocity components relative to the earth along

*This section was added by Lin, after the rest of the paper had been completed. For related discussions of vortex motions in the atmosphere, see a recent paper by Rossby [7]. 
the directions of increasing $\varphi$ and $\sigma$, and let $\zeta$ be the component of absolute vorticity in the direction of the $z$-axis. Then the vorticity equation

$$
\frac{\partial \zeta}{\partial t}+\frac{u}{r} \frac{\partial \zeta}{\partial \varphi}+v \frac{\partial \zeta}{\partial \sigma}=0
$$

where $r$ is the distance from the axis of rotation, and

$$
\zeta=\zeta_{n}+\frac{1}{r}\left\{\frac{\partial v}{\partial \varphi}-\frac{\partial}{\partial \sigma}(r u)\right\}
$$

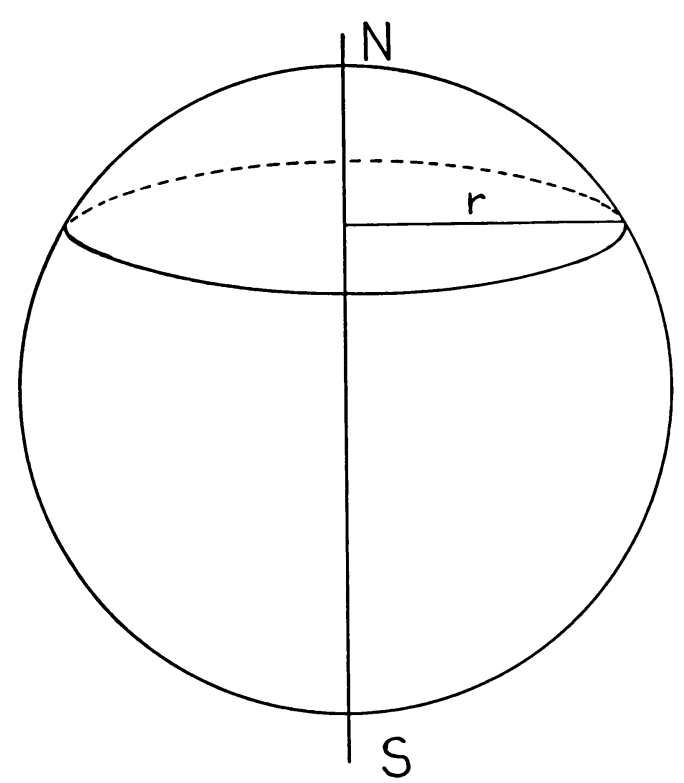

Fig. 4.

with $\zeta_{n}$ as the component of vorticity of spinning normal to the surface of the earth. The equation of continuity is

$$
\frac{\partial u}{\partial \varphi}+\frac{\partial}{\partial \sigma}(r v)=0 .
$$

Let us consider a motion slightly disturbed from a pure zonal motion

$$
u=u_{0}(\sigma), \quad v=0 .
$$

The perturbations $u^{\prime}, v^{\prime}$ and $\zeta^{\prime}$ then satisfy the linearized equations

$$
\begin{aligned}
& \frac{\partial u^{\prime}}{\partial \varphi}+\frac{\partial}{\partial \sigma}\left(r v^{\prime}\right)=0 \\
& \frac{\partial \zeta^{\prime}}{\partial t}+\frac{u_{0}}{r} \frac{\partial \zeta^{\prime}}{\partial \varphi}+v^{\prime} \frac{d \zeta_{0}}{d \sigma}=0,
\end{aligned}
$$


with

$$
\begin{aligned}
\zeta_{0} & =\zeta_{n}-\frac{1}{r} \frac{d}{d \sigma}\left(r u_{0}\right) \\
\zeta^{\prime} & =\frac{1}{r}\left\{\frac{\partial v^{\prime}}{\partial \varphi}-\frac{\partial}{\partial \sigma}\left(r u^{\prime}\right)\right\} .
\end{aligned}
$$

It is now possible to set up an "equivalent" plane motion by defining the following quantities:

$$
\begin{aligned}
& x_{*}=\varphi, \quad y_{*}=\int_{0}^{\sigma} \frac{d \sigma}{r}, \quad w_{*}=\frac{u_{0}}{r} \\
& u_{*}^{\prime}=r u^{\prime}, \quad v_{*}^{\prime}=r v^{\prime}, \quad \zeta_{*}^{\prime}=r^{2} \zeta^{\prime} .
\end{aligned}
$$

Then

$$
\begin{aligned}
& \frac{\partial u_{*}^{\prime}}{\partial x_{*}}+\frac{\partial v_{*}^{\prime}}{\partial y_{*}}=0 \\
& \frac{\partial \zeta_{*}^{\prime}}{\partial t}+w_{*} \frac{\partial \zeta_{*}^{\prime}}{\partial x_{*}}+v_{*}^{\prime} \frac{d \zeta_{0}}{d y_{*}}=0 \\
& \zeta_{*}^{\prime}=\frac{\partial v_{*}^{\prime}}{\partial x_{*}}-\frac{\partial u_{*}^{\prime}}{\partial y_{*}}
\end{aligned}
$$

Thus, if we now drop the asterisks the problem discussed in Sec. 3 is reproduced with

$$
g=\frac{d \zeta_{0}}{d y}
$$

The physical interpretation of the quantity $\tau$ defined by (12) is also obvious at once. It is $(1 / 2 \pi)$ times the total transfer of angular momentum per unit height across the latitude circle of radius $r$.

The disturbance equation for the spherical surface has been obtained and briefly discussed by Kuo.

Most of the results obtained in Secs. 3, 4 are applicable to the present case. There are, however, also essential differences. In the first place, the final solution has a meaning only when $\alpha$ is an integer. During the investigation of the eigenvalue problem, however, we shall of course investigate all positive values. Another essential modification is the following. Since $g \neq-w^{\prime \prime}$, the existence proof of Sec. 4 cannot be readily extended to the present case. It is also clear that the symmetry or asymmetry of the velocity distribution is not an essential feature. Rather the equality or inequality of the values of $w$ at the roots of $g=0$ is what distinguishes cases (a) and (b) in Sec. 4.

The details of the investigation are also modified because the velocity distribution is more complicated. Typical diagrams of $w(y)$ and $g(y)$ are shown in Fig. 5. It is clear that one may consider only half of the flow (say right), and that the boundary conditions are

$$
\varphi=0, \quad \text { or } \quad \varphi^{\prime}=0, \quad \text { at } \quad y=0 \text {, }
$$

and

$$
\varphi \rightarrow 0, \quad \varphi^{\prime} / \varphi=-\alpha \text { in the limit as } y \rightarrow \infty \text {. }
$$


But since these conditions give $\tau=0$ at both ends, no essential modification is necessary. The diagrams for the distribution of $\tau$ are similar to those shown in Sec. 4. In particular, one notes that the angular momentum is transferred northwards by a neutral disturbance if $w_{s}<w_{t}$ (opposite to the case shown in Fig. 5), i.e., if the mean zonal wind at the point of minimum vorticity is lower than that at maximum vorticity. Otherwise, a damped disturbance is necessary for northward transfer of angular momentum.
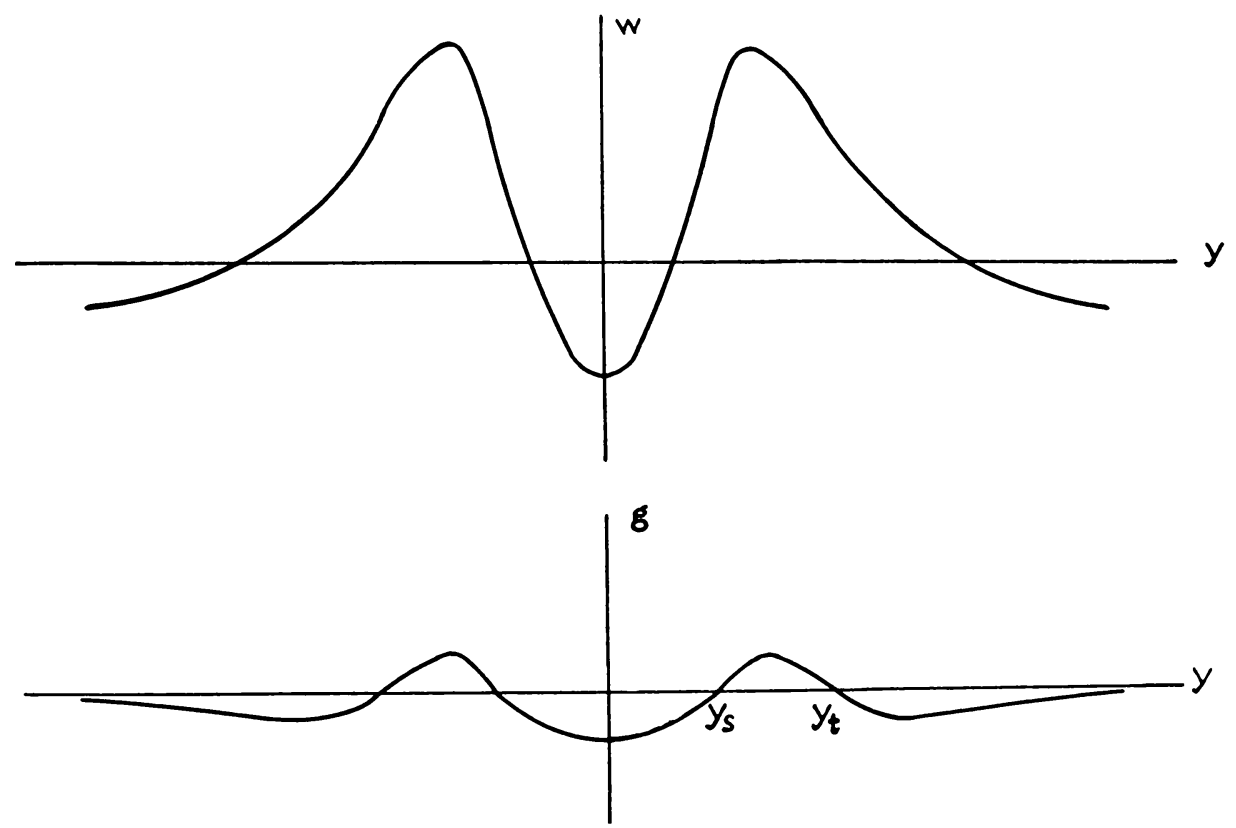

FIG. 5.

The existence of self-excited and neutral disturbances will now be discussed for the typical velocity and vorticity distribution shown in Fig. 5. We shall consider essentially the neutral disturbance in the case $w_{s}=w_{t}$, because the other cases can be covered by arguments similar to those used in Sec. 4 . For this case, with $c=w_{s}=w_{t}$,

$$
K \equiv \frac{g}{w-c}
$$

is a regular non-negative function which approaches zero as $y \rightarrow \pm \infty$. Consider only $y>0$. The equation

$$
\varphi^{\prime \prime}+\lambda \varphi+K \varphi=0
$$

obtained from (9a) with $-\alpha^{2}$ replaced by $\lambda$, must now be shown to have a negative eigenvalue. Now, the first eigenvalue is [9]

$$
\lambda_{1}=\min . \frac{\int_{0}^{\infty}\left(f^{\prime 2}-K f^{2}\right) d y}{\int_{0}^{\infty} f^{2} d y}
$$


for all functions $f$ having proper differentiability properties, and satisfying the boundary condition $f^{\prime}(0)=0$ and making $\int_{0}^{\infty} f^{2} d y$ convergent. We want to show that for certain functions satisfying the boundary conditions the integral in the numerator is negative, for then $\lambda_{1}$ is negative and our result is proved.* For this purpose, take $f=e^{-c y^{2}}$. Then

$$
\int_{0}^{\infty}\left(f^{\prime 2}-K f^{2}\right) d y=\int_{0}^{\infty}\left(4 \epsilon^{2} y^{2}-K\right) e^{-2 \epsilon y^{2}} d y .
$$

As $\epsilon \rightarrow 0$, this integral approaches $-\int_{0}^{\infty} K d y$. Hence, by taking some small but finite value for $\epsilon$, the integral can be made negative, completing our proof.

6. Validity of the asymptotic expansions. We shall now slightly extend some theorems of Wasow on the asymptotic solutions of the equation of hydrodynamic stability of two-dimensional parallel flows. Wasow established the validity of the formal expansions in the neighborhood of a single critical point. We shall note the modifications necessary to make the theorems of Wasow applicable to cases with two critical points. The preceding sections have used the mathematical justification so derived.

We shall retain the notation of Wasow in the main, but with $x_{0}, x_{0}^{*}$ for the two isolated zeros; $A_{k}, A_{k}^{*}, k=1,2,3$, for the exponential-type solutions; and so on (see Fig. 6).

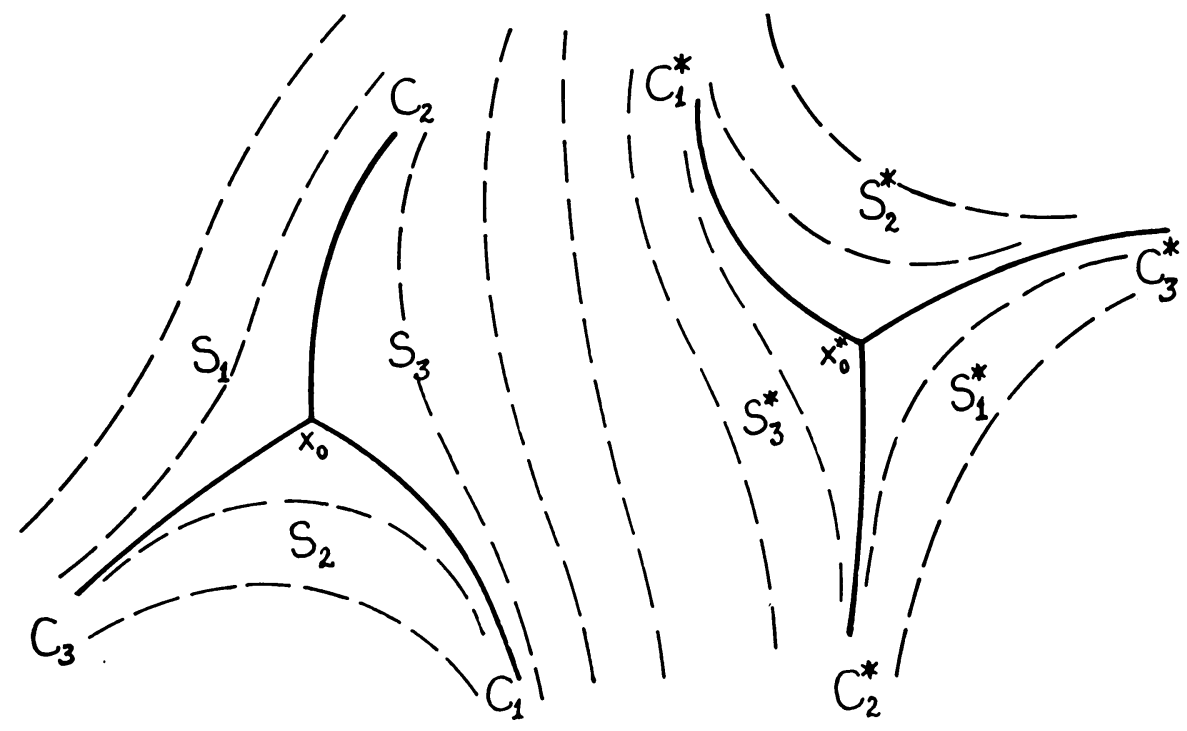

FIG. 6.

The jet flow profiles require the assumption of two zeros for the function $b_{0}(x)$ in the complex $x$-plane and further require that the region of validity of the asymptotic expressions have indefinite extent in order that the real $x$-axis may be used for certain integrals.

The following lemma is proved by Wasow, p. 857:

*This proof is similar to the one used by Friedrichs [6]; but the function $f(y)$ has to be chosen differently here. 
LEMMA. Let $T^{*}$ be a subdomain of $T$ every point of which can be reached from a fixed point $p$ by a path $\Gamma$ in $T$ along which the $\tau n$ quantities

$$
\operatorname{Re}\left(P_{i}-P_{i}\right), \quad(i=1,2, \cdots, \tau ; j=1,2, \cdots, n),
$$

are monotonically non-decreasing. Then there exist $\tau$ linearly independent solutions $y=$ $y_{r}(x, \lambda), r=(1,2, \cdots, \tau)$ of (12) for which

$$
y_{r}(x, \lambda)=e^{P_{r}(x, \lambda)}\left[\sum_{\nu=0}^{\nu_{2}} S_{r \nu}(x) \lambda^{-\nu / k}+E\left(T^{*}\right) \lambda^{-(\nu,+1) / k}\right],
$$

where $\nu_{2}$ is an integer depending on the choice of $\nu_{1}$, and $\lim _{n \rightarrow \infty} \nu_{2}=\infty$.

Apparently to obtain a definite region of validity for the existence proof, Wasow identifies $T$ with the doubly connected domain $S$, and $T^{*}$ with $S-C_{k}$. However, the only special property required of the domain of validity, whatever its shape may be, is the monotonicity condition of the Lemma. By examining the lines $\operatorname{Re}[\lambda Q(x)]=$ const. and $\operatorname{Re}\left[\lambda Q^{*}(x)\right]=$ const. we can easily identify $T$ and $T^{*}$ with regions suitable for our purposes.

In view of the equation

$$
Q(x)=Q\left(x_{0}^{*}\right)+Q^{*}(x),
$$

it is easy to show that the necessary and sufficient condition that no $C_{k}$ intersects any $C_{k}^{*}$ is

$$
\operatorname{Re}\left[\lambda Q\left(x_{0}^{*}\right)\right] \neq 0 .
$$

This condition will be assumed since it holds for any reasonable velocity profile. Then one sector at $x_{0}$ has a common part with one sector at $x_{0}^{*}$, and by (34) the pair of curves bounding this domain and starting from $x_{0}^{*}$ belong to the family

$$
\operatorname{Re}[\lambda Q(x)]=\text { constant, }
$$

and indeed the constant is $\pm \operatorname{Re}\left[\lambda Q\left(x_{0}^{*}\right)\right]$. Similarly the other pair of bounding curves belongs to the family

$$
\operatorname{Re}\left[\lambda Q^{*}(x)\right]=\text { constant. }
$$

A set of contour lines (36) and (37) then fills the $x$-plane (Fig. 6) in an entirely regular manner except at $x_{0}$ and $x_{0}^{*}$; i.e., through each point is one and only one curve and any finite region containing neither $x_{0}$ nor $x_{0}^{*}$ nor points of more than two sectors at $x_{0}$ and two sectors at $x_{0}^{*}$ is mapped one-to-one to the corresponding region of the $\lambda Q(x)$ plane. For example, regions $S_{2}, S_{3}, S_{2}^{*}, S_{3}^{*}$ are mapped as shown in Fig. 7. In this case the boundaries $C_{3}, C_{2}$ map into the cut $C_{0}$ through the origin, and $C_{2}^{*}, C_{3}^{*}$ map into $C_{0}^{*}$. Any path $\Gamma$ of importance here passes through the common sector $S_{3}, S_{3}^{*}$ and we may take it to pass also into $S_{2}$ and $S_{2}^{*}$ for definiteness. Then if we have chosen $\operatorname{Re}[\lambda Q(x)]<0$ in $S_{2}$, on crossing $C_{1}$ we have $\operatorname{Re}[\lambda Q(x)]>0$ on the remainder of $\Gamma$. This holds also if $S_{1}^{*}$ is used in place of $S_{2}^{*}$. It is clear from the geometrical configuration that any two points in the plane can be joined by a path $\Gamma$ on which $\operatorname{Re}[\lambda Q(x)]$ is monotonically nondecreasing, where we select the subdominant determination of the real part of $\lambda Q(x)$ at the point $p$ of the Lemma. This is essentially what Wasow does in choosing the point $p_{k}$ on the boundary arc $B_{k}$ of the sector $S_{k}$ where $\operatorname{Re}[\lambda Q(x)]$ takes on its minimum. 
The coefficients of the basic differential equation may be taken to be such that the coefficients in the series (10), page 857 of [4], are regular analytic in the entire plane so that $T$ can be identified with the plane, while $T^{*}$ can be identified with any one of an infinite set of finite regions satisfying both the monotonicity property and the property

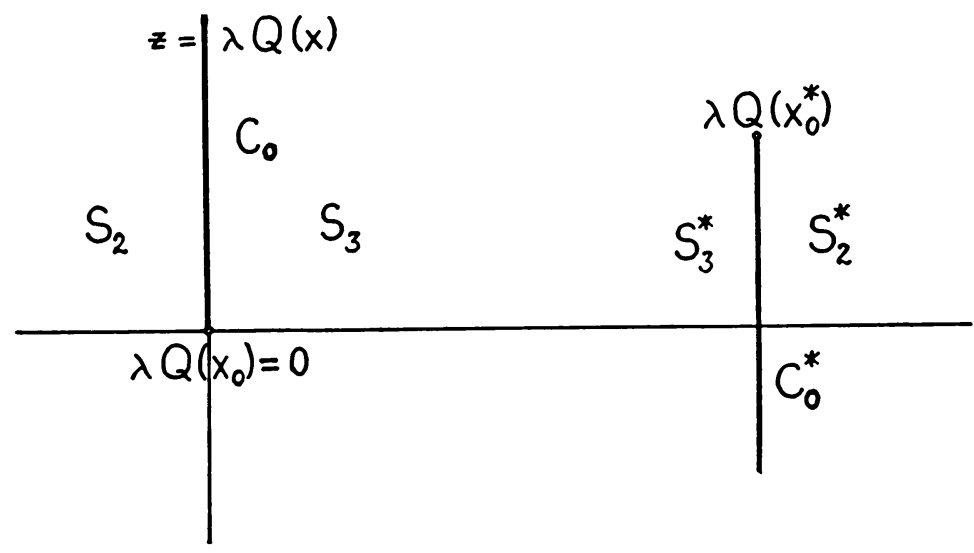

Fig. 7.

that the modulus of $x$ can be taken indefinitely large. This is sufficient for the asymptotic expansions since $x$ will be fixed at some finite point while $|\lambda| \rightarrow \infty$.

Then the theorems of Wasow follow formally by omitting the domain $S$ from consideration and replacing it by any finite region of the above type.* In Sec. 2 we used this result with the following change of notation: $x$ replaced by $y ;-b_{0}(x)$ by $w(y)-c$; and $\left\{V, U_{1}, A_{3}, A_{2}\right\}$ by $\left\{\varphi_{1}, \varphi_{2}, \varphi_{3}, \varphi_{4}\right\}$ respectively.

*A different proof was given in the thesis, using only the final theorems of Wasow.

\section{REFERENCES}

1. C. C. Lin, On the Stability of Two-Dimensional Parallel Flows, Parts I, II, III; Q. Appl. Math. 3, 117142, 218-234, 277-301 (1945).

2. M. Lessen, On the Stability of the Free Laminar Boundary Layer between Parallel Streams, Thesis for Sc.D. at Mass. Inst. of Tech. (1948); also NACA Tech. Note No. 1929 (1947).

3. P. Chiarulli, Stability of Two-dimensional Velocity Distributions of the Half-Jet Type, Thesis for Ph.D. at Brown Univ. (1949); also Headquarters Air Material Command Technical Report No. F-TS-1228-IA (1949).

4. W. Wasow, The Complex Asymptotic Theory of a Fourth Order Differential Equation of Hydrodynamics, Annals Math., 49, 852-871 (1948).

5. W. Tollmien, Ein allgemeines Kriterium der Instabilitaet laminarer Geschwindigkeitsverteilungen, Nachr. Ges. Wiss. Gottingen (neue Folge), 1, 79-114 (1935).

6. R. von Mises and K. O. Friedrichs, Fluid Dynamics, Brown University Notes, pp. 205-207 (1941).

7. C. G. Rossby, On a Mechanism for the Release of Potential Energy in the Atmosphere, Jour. of Meteorology, 6, 163-180 (1949).

8. Hsiao-lan Kuo, Dynamic Instability of Two-Dimensional Nondivergent Flow in a Barotropic Atmosphere, Jour. of Meteorology, 6, 105-122 (1949).

9. R. Courant and D. Hilbert, Methoden der Mathematischen Physik, vol. 1, p. $344 \mathrm{ff}$. 